\title{
Keyword index
}

achiasmatic meiosis $\mathbf{6 4 5}$

Actinidia $\mathbf{3 2 8}$

Ag staining 229

Ag-NORs $\mathbf{5 7 8}$

alcohol dehydrogenase $\mathbf{1 0 1}$

Allium cepa $\mathbf{4 0 3}$

allozymes $41,57,84,101,166,241,293,311,363,445$,

470, 476, 552, 590, 665

Ambystoma tigrinum 101

androdioecy $\mathbf{5 0 , 1 9 0}$

antagonistic pleiotropy $\mathbf{5 0 7}$

ants $\mathbf{5 7 8}$

APRT 21

Atlantic salmon $\mathbf{6 2 0}$

autotroph vs. heterotroph 498,507

Avena sativa 115

B1/B2 elements 21

B chromosomes $\mathbf{6 3 6}$

B transmission control $\mathbf{6 3 6}$

Bactrocera cucurbitae $\mathbf{9 3}$

Beringia 363

biodiversity $\mathbf{5 3 9}$

birth-death process $\mathbf{4 1 7}$

bivalent orientation $\mathbf{5 6 1}$

bracket crossing $\mathbf{5 7 1}$

breeding system 190

broad-sense heritability

124

broomrape $\mathbf{6 8}$

Bryophyta 293

butterfly mimicry $\mathbf{6 2 8}$

Callosobruchus maculatus $\mathbf{1 5 8}$

Camellia spp. $\mathbf{6 0 3}$

cave crickets $\mathbf{6 6 5}$

Cetaceae $\mathbf{2 8 4}$

chiasma terminalization $\mathbf{5 6 1}$

chiasmata $\mathbf{5 1 5}$

Chlamydomonas $\quad 490,498,507$

chromosome elimination 464

clonal heterogeneity $\mathbf{6 1 2}$

clonal plant 293

clonal reproduction $\mathbf{5 7}$

Coast redwood 166

colonizing species $\mathbf{2 6 1}$

confidence interval $\mathbf{3 4 5}$

conservation genetics 311, 417,

correlated response $\mathbf{4 9 8}$ cost of adaptation $\mathbf{4 9 8}$

Cyclamen $\mathbf{2 0 5}$

cyclical parthenogenesis $\mathbf{1 8 2}$

cytogenetics 612

cytoplasmic incompatibility $\mathbf{3 9 1}$

cytoplasmic male sterility $\mathbf{7 5}$

D-loop 284

Danish waters $\mathbf{2 7 0}$

Daphnia 182, 363

Datisca glomerata 190

Datiscaceae 190

developmental instability $\mathbf{1 8 2}$

developmental stability $\mathbf{2 1 5}$

differentiation $\mathbf{4 7 6}$

dioecy $\mathbf{5 0}$

discriminant analysis $\mathbf{4 7 0}$

disequilibrium 476

disomic inheritance $\mathbf{3 2 8}$

dispersal $\mathbf{5 7}$

diversity 68,403

DNA fingerprinting $\mathbf{4 5 7}$

DNA profile $\mathbf{5 8 3}$

drift 101

Drosophila 302, 354

D. buzzatii $\mathbf{4 1 0}$

D. melanogaster 1

edaphic ecotype $\mathbf{5 5 2}$

electrophoresis $\mathbf{6 5 5}$

elongation factor $\mathbf{1}$

embryos $\mathbf{4 1 0}$

endemic species $\mathbf{3 1 1}$

environmental heterogeneity $\mathbf{4 9 0}$

environmental sensitivity 124

Epilachna pustulosa $\mathbf{2 7 7}$

epistatic effect $\mathbf{3 5 4}$

Eucalyptus $\mathbf{2 5 2}$

evolution 235, 284

evolution of plasticity $\mathbf{5 0 7}$

evolvability 261

experimental evolution $\mathbf{4 9 0}$

extinction $\mathbf{3 1 1}$

Fagus crenata 241

fecundity 93

Festuca arundinacea 464

Feulgen densitometry $\mathbf{5 4 7}$ 


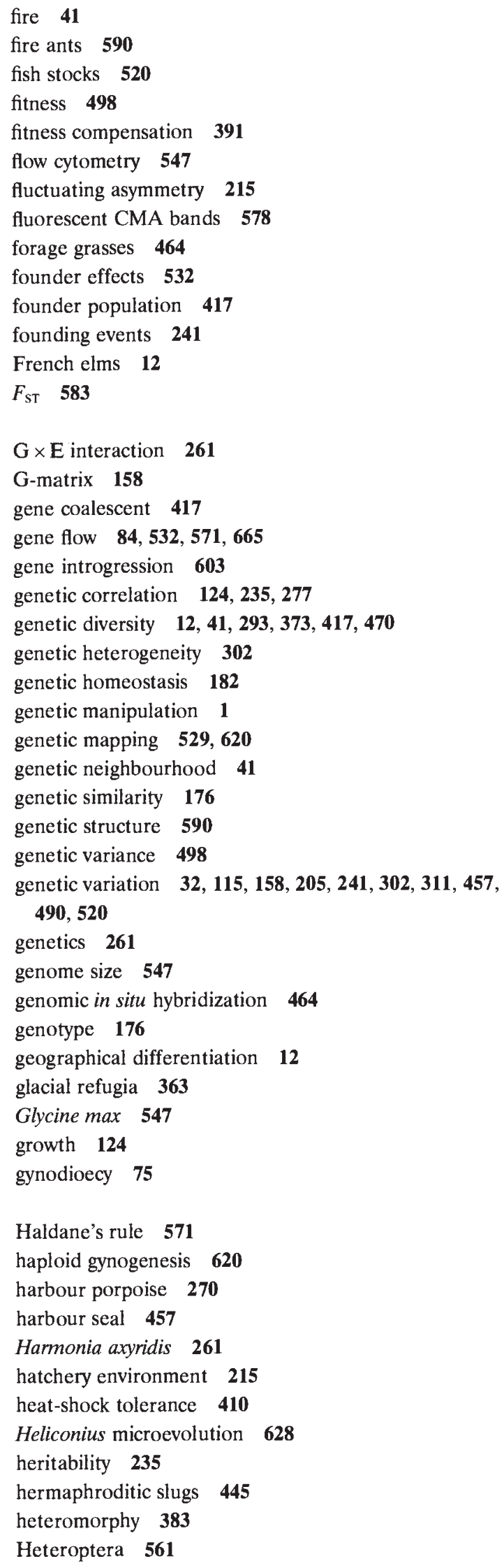

heterozygote deficiency $\mathbf{5 7 1}$

heterozyosity $\mathbf{2 1 5}$

hierarchical variation $\mathbf{2 9 3}$

holokinetic chromosomes $\mathbf{5 6 1}$

homozygous strains $\mathbf{4 4 5}$

Hordeum spontaneum $\mathbf{3 7 3}$

host plants $\mathbf{2 7 7}$

host-plant adaptation $\mathbf{8 4}$

hybrid zone $\mathbf{5 7 1}$

hybridization $\mathbf{2 5 2}$

hybrids 12

Hylocomium splendens 293

hypoxia 101

in situ hybridization $\quad 229,529,539,578$

inbreeding 182, 410

inbreeding depression $\mathbf{2 5 2}$

incompatibility $\mathbf{3 8 3}$

inheritance 166

interval mapping 146

inverted meiosis $\mathbf{5 6 1}$

islands $\mathbf{3 1 1}$

isolation by distance $\mathbf{5 3 2}$

isozymes $12, \mathbf{3 2 8}, \mathbf{5 2 0}, \mathbf{5 3 2}, \mathbf{6 5 5}$

kiwifruit 328

landraces $\mathbf{5 3 9}$

late-acting self-sterility $\mathbf{1 3 5}$

lethal gene $\mathbf{3 8 3}$

lichens $\mathbf{3 7 3}$

lifespan 1

linkage 645

local asymptotic framework $\mathbf{3 4 5}$

Locusta migratoria $\mathbf{5 1 5}$

Lolium multiflorum 464

longevity 93

low nucleotide diversity $\mathbf{2 8 4}$

maize 146

male-killing 391

mapping of allozymes 645

marginal populations $\mathbf{5 5 2}$

mathmatical model 391

mating system 205

maturity group $\mathbf{5 4 7}$

meiosis $\mathbf{6 3 6}$

Metropolis algorithm $\mathbf{5 8 3}$

microgametogenesis $\mathbf{6 3 6}$

microgeographical structure $\mathbf{3 0 2}$

microgeographical variation $\mathbf{3 7 3}$

microsatellites $\mathbf{2 7 0 , 6 2 0}$

mimicry evolution 628

molecular differentiation $\mathbf{4 3 3}$

(C) The Genetical Society of Great Britain, Heredity, 78, 683-686. 
$\begin{array}{lr}\text { Morone } & \mathbf{3 2} \\ \text { mouse } & \mathbf{4 2 4}\end{array}$

mtDNA variation $\mathbf{7 5}, \mathbf{3 6 3}, 590$

Müllerian mimicry 628

mutation accumulation $\mathbf{5 0 7}$

Narcissus triandrus $\mathbf{1 3 5}$

neutral theory $\mathbf{6 5 5}$

niche overlap $\mathbf{1 7 6}$

norm of reaction $\mathbf{1 7 6}$

novel environment 158

nucleolar organizing regions $\mathbf{2 2 9}$

nucleotide divergence $\mathbf{4 3 3}$

oaks 433

oats 115

Odontocetae $\mathbf{2 8 4}$

onion $\mathbf{4 0 3}$

Orthoptera $\mathbf{5 1 5}$

ostracod $\mathbf{5 7}$

outcrossing rates $\mathbf{1 3 5}, \mathbf{2 5 2}$

parthenogenesis $\mathbf{5 7}$

Pennisetum $\mathbf{5 2 9}$

phenetics 603

phenotypic plasticity $\quad$ 176, 261

phylogeny 21

phylogeography 363

physcial mapping $\mathbf{5 2 9}$

Picea glehnii $\mathbf{4 7 0}$

plant breeding $\mathbf{5 3 9}$

Plantago lanceolata $\mathbf{7 5}$

pleiotropy $\mathbf{9 3}$

Poecilia formosa $\mathbf{6 1 2}$

pollen 146

pollen mitosis $\mathbf{6 3 6}$

polyploid 166,328

population differentiation 205,241

population expansion $\mathbf{2 4 1}$

population extinction 41

population genetics $32,270,284,445,470,583,628,665$

population size $\mathbf{5 5 2}$

population structure $\mathbf{5 7 , 8 4}$

Populus 124

position effects 1

Primula $\mathbf{3 8 3}$

Primulaceae $\mathbf{2 0 5}$

QTL location 345

QTL $\times$ environment interaction $\mathbf{1 4 6}$

Quercus petraea $\mathbf{4 7 6}$

RAPD $32,68,403,433,603$

RAPD-PCR 176 rapid microevolution $\quad 628$

rate variation $\mathbf{2 1}$

rDNA 115, 529

reaction norms $\mathbf{2 7 7}$

recolonization $\mathbf{4 1}$

recombination 383,515

relative rate test $\mathbf{2 1}$

reproductive assurance $\mathbf{5 0}$

reproductive strategy $\mathbf{4 0 3}$

resource subdivision $\mathbf{3 0 2}$

RFLPs 146, 532

ribosmoal intergenic spacer $\mathbf{1 1 5}$

ribosomal cistrons $\mathbf{2 2 9}$

rodent 21

rye $\mathbf{5 3 9}$

Salmo salar $\mathbf{2 1 5 , 6 2 0}$

sardine $\mathbf{5 2 0}$

SCAR 433

sea beet $\mathbf{5 3 2}$

Secale cereale $\mathbf{6 3 6}$

seed abortion $\mathbf{2 5 2}$

segregation analysis $\mathbf{4 2 4}$

selection 93, 101, 373, 490

selection experiment 498,507

selective coefficients $\mathbf{6 5 5}$

self-fertilization $\mathbf{2 5 2}$

sensitivity 424

Sequoia sempervirens $\mathbf{1 6 6}$

sex change $\mathbf{5 0}$

sex choice $\mathbf{5 0}$

sex chromosome trivalent $\mathbf{6 4 5}$

sex determination 190

sexual dimorphism $\mathbf{5 0}$

sexual polymorphism 135

shallot $\mathbf{4 0 3}$

Silene nutans $\mathbf{5 5 2}$

similar test $\mathbf{3 4 5}$

similarity 603

size effects 1

social organization $\mathbf{5 9 0}$

Solenopsis invicta $\mathbf{5 9 0}$

somatic recombination $\mathbf{4 6 4}$

speciation $\mathbf{3 5 4}$

species differences 354

sperm length 354

stochastic demography $\mathbf{4 1 7}$

stress $\mathbf{4 1 0}$

striped bass $\mathbf{3 2}$

supergene $\mathbf{3 8 3}$

sympatry 205

synaptonemal complex $\mathbf{5 1 5}$

testis length $\mathbf{3 5 4}$

(c) The Genetical Society of Great Britain, Heredity, 78, 683-686. 
686 KEYWORD INDEX

tetrasomic inheritance $\mathbf{3 2 8}$

trade-off 93, 158, 507

tristyly 135

trypanotolerance $\mathbf{4 2 4}$

\section{Ulmus 12}

uniparental reproduction $\mathbf{4 4 5}$

unisexual fish 612 water quality $\mathbf{2 1 5}$

waterstrider $\mathbf{5 7 1}$

West Greenland 270

wheat 539

wild barley $\mathbf{3 7 3}$

wing dimorphism 235

Wolbachia 391 